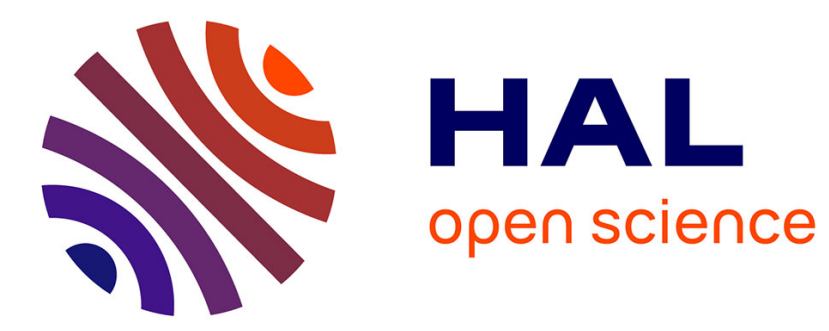

\title{
Les processus de néolithisation en Italie septentrionale.
} Thomas Perrin

\section{To cite this version:}

Thomas Perrin. Les processus de néolithisation en Italie septentrionale.. Bulletin de la Société préhistorique française, 2004, 101 (4), pp.887-891. hal-00266609

\section{HAL Id: hal-00266609 https://hal.science/hal-00266609}

Submitted on 1 Nov 2018

HAL is a multi-disciplinary open access archive for the deposit and dissemination of scientific research documents, whether they are published or not. The documents may come from teaching and research institutions in France or abroad, or from public or private research centers.
L'archive ouverte pluridisciplinaire HAL, est destinée au dépôt et à la diffusion de documents scientifiques de niveau recherche, publiés ou non, émanant des établissements d'enseignement et de recherche français ou étrangers, des laboratoires publics ou privés. 


\section{CORRESPONDANCE SCIENTIFIQUE}

\section{Les processus de néolithisation en Italie septen- trionale}

Le passage du mode de vie des chasseurs - collecteurs à celui des paysans - éleveurs a été l'un des bouleversements fondamentaux de l'histoire de l'humanité. Ce changement a non seulement touché le monde matériel, par le passage à l'économie de production, mais il a également fortement modifié l'organisation même des sociétés, tant d'un point de vue social que spirituel (cf. par exemple Cauvin, 1994). Pour l'Europe, l'origine de ces transformations se place dans un foyer proche-oriental, vers le $\mathrm{IX}^{\mathrm{e}}$ millénaire, à partir duquel ce monde néolithique va ensuite diffuser pour atteindre peu à peu l'ensemble de l'Europe occidentale. Si l'on pouvait auparavant penser que cette néolithisation avait pour principal vecteur le déplacement physique de groupes de colons, dont on pouvait d'ailleurs estimer la "vague d'avance" (Ammerman et Cavalli Sforza, 1971), toutes les recherches récentes tendent à montrer, au contraire, que la néolithisation fut un phénomène extrêmement complexe et de longue durée. À l'image de cette "vague d'avance" succède aujourd'hui celle d'une progression "arythmique" (Guilaine, 2000, p. 15) et le scénario de colonisation se complète de phénomènes plus complexes de diffusion, d'acculturation des populations de chasseurs mésolithiques autochtones (cf. par exemple Mazurié de Keroualin, 2003 pour une présentation de ces questions à l'échelle de l'Europe). Aujourd'hui, on ne peut plus aborder le phénomène de néolithisation sans chercher à comprendre le rôle qu'ont pu y tenir les dernières sociétés de chasseurs - collecteurs. Malheureusement, pour la partie sud occidentale de l'Europe, qui nous occupera plus particulièrement ici, force est de constater le très fort manque de données concernant le Mésolithique récent/final, période correspondant grosso modo au $\mathrm{VI}^{\mathrm{e}}$ millénaire. Dans quelques régions cependant, cette période est mieux documentée et il est alors possible de travailler sur les scénarios d'interactions entre les divers groupes culturels impliqués dans les processus de néolithisation (pour une réflexion générale, cf. par ex. Zvelebil, 2000). C'est par exemple le cas en Espagne, dans le pays Valencien, où les chercheurs ont montré l'existence de liens entre agriculteurs néolithiques et chasseurs mésolithiques au sein d'un modèle dit "dual" (Juan Cabanilles, 1990). Une probable coexistence territoriale à l'origine de contacts et d'échanges entre ces deux mondes a également été montrée en France dans la vallée du Rhône (Perrin, 2003). Ces phénomènes de coexistence territoriale amenant à des interactions culturelles sont aussi attestés au Portugal (Marchand, 2001). En Italie, alors que le Mésolithique récent/final est quasi absent dans toute une grande moitié sud de la péninsule, il est mieux connu au nord, notamment dans la vallée de l'Adige, où les caractères originaux de certains groupes du Néolithique ancien, comme le groupe de Gaban, sont mis sur le compte de l'acculturation (la "céramisation") plus ou moins forte de ces derniers groupes de chasseurs (Bagolini et Biagi, 1988). Dans le cadre d'une réflexion plus générale sur la néolithisation de l'Italie du Nord, financée grâce à l'obtention d'une bourse de recherches postdoctorales de la Fondation Fyssen, il m'a paru important d'essayer de renouveler le cadre de compréhension de ces processus par l'étude des productions en silex taillés des diverses populations impliquées. Les outils de pierre taillée présentent en effet cet avantage d'être bien représentés non seulement chez les populations de chasseurs collecteurs mésolithiques mais aussi chez les groupes d'agriculteurs éleveurs néolithiques. En appréhendant ces outillages par le biais d'une analyse "techno-économique", il est alors possible de reconstituer les schémas opératoires, conceptuels, des artisans producteurs, et par là, de remonter le fil des traditions culturelles ou des ruptures.

\section{Les groupes culturels impliqués dans la néolithisation} du Nord-Est de l'Italie

Avant de présenter les résultats de cette recherche, il me paraît utile de brosser ici une brève présentation des divers groupes culturels impliqués dans la néolithisation 


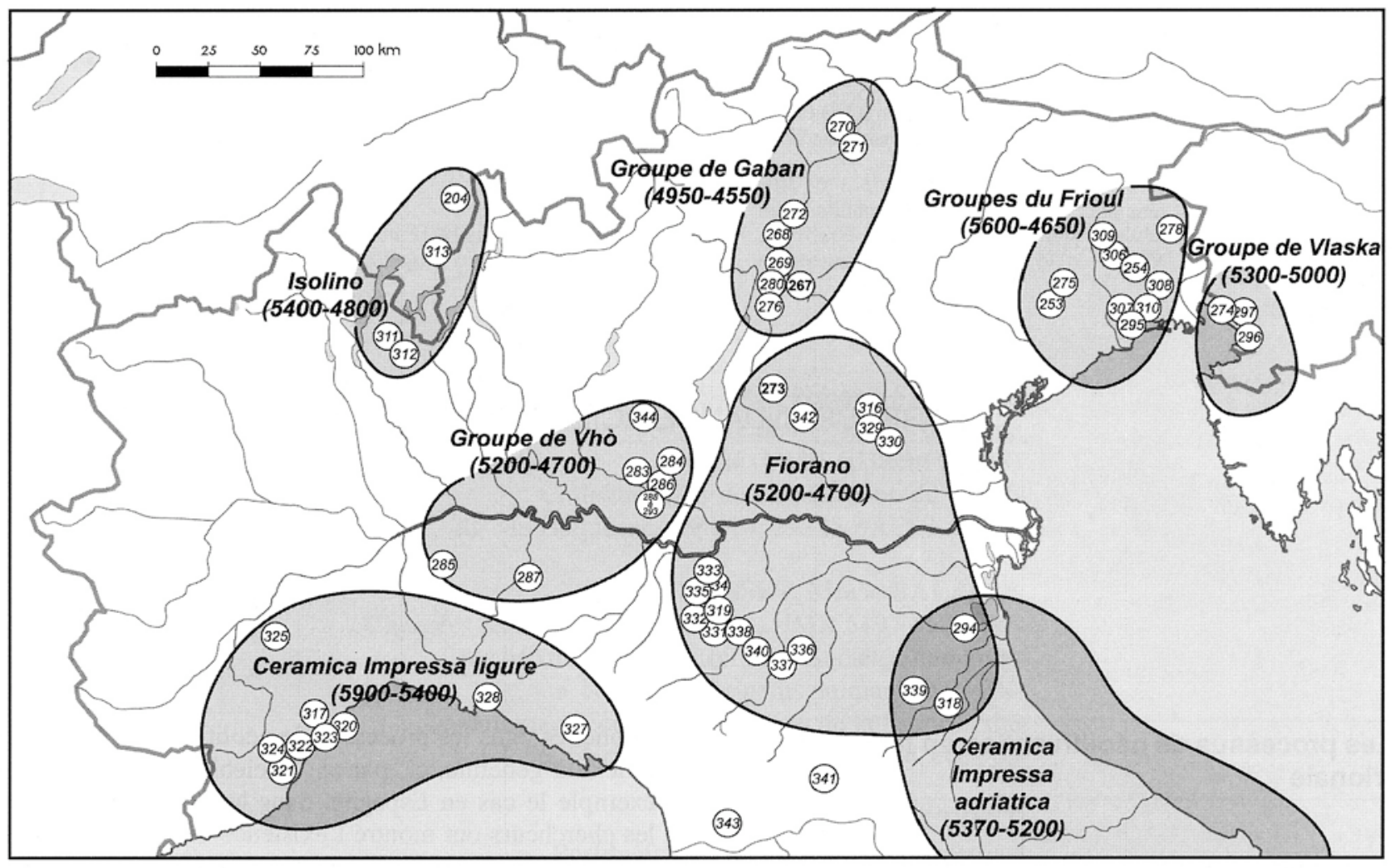

Fig. 1 - Carte de situation des principaux sites et groupes culturels impliqués dans le processus de néolithisation de l'Italie septentrionale. Les dates indiquées sont exprimées en années solaires, avant notre ère. L'abri Gaban (Trento), dont il question dans le texte, est représenté par le numéro 267.

de cette partie de l'Italie, la situation y étant relativement complexe (fig. 1).

Les derniers groupes de chasseurs - collecteurs mésolithiques se rattachent au Castelnovien. Cette culture se développe sur quasiment l'ensemble de l'Europe sud occidentale à partir du milieu du $\mathrm{VII}^{\mathrm{e}}$ millénaire environ jusqu'à la fin du $\mathrm{VI}^{\mathrm{e}}$, en rupture complète d'avec les traditions culturelles antérieures, sans que l'on ne comprenne d'ailleurs réellement ni l'origine ni le moteur de ce changement radical.

Dans la plaine padane, le premier néolithique est représenté par les groupes de Vhò et de Fiorano. Si le premier se développe surtout dans la Crémone et le second plutôt dans le Véronèse, ils sont en réalité relativement proches l'un de l'autre, d'un point de vue tant culturel que chronologique (5200-4700 avant notre ère ; leur individualisation traduit en fait plus la structure administrative de la recherche en Italie que la réalité archéologique). Ces deux groupes sont généralement considérés comme résultant d'une extension vers le nord de la culture de la Ceramica Impressa adriatica, laquelle représente le plus ancien néolithique de la frange adriatique de l'Italie centrale, mâtinée d'influences balkaniques.

Ces influx orientaux atteindraient d'ailleurs la plaine du Pô à travers le karst de Trieste et la Vénétie, où se développent respectivement le groupe de Vlaska et les groupes du Frioul. Le premier est une émanation côtière du Néolithique ancien balkanique. Il se développerait entre 5300 et 5000 , mais reste en réalité actuellement très mal connu, essentiellement par des fouilles anciennes. Les groupes du Frioul se placent eux entre 5600 et 4650 avant notre ère et comportent deux faciès : celui de Fagnigola et celui de Sammardenchia, du nom des deux sites éponymes.

Enfin, le groupe de Gaban occupe le Val d'Adige uniquement, entre 4950 et 4550 et est considéré comme issu de l'acculturation de groupes mésolithiques par les premiers néolithiques. La pertinence de cette hypothèse est un point fondamental dans l'appréhension des processus de néolithisation puisqu'il s'agirait là d'un des rares exemples de transfert, où des chasseurs abandonneraient une partie de leurs traditions techniques pour acquérir celles de groupes néolithiques.

\section{Le groupe de Gaban, un faciès mixte mésolithique - néolithique?}

L'abri-sous-roche de Gaban se trouve à proximité même de la ville de Trento ( $\mathrm{n}^{\circ} 267$ sur la fig. 1), et a été fouillé dans les années 1970-1980 par B. Bagolini, alors professeur à l'université de Trento. La découverte de "couches" comportant des industries lithiques de traditions mésolithiques associées à de la céramique du premier Néolithique (influences du Fiorano et des groupes du Frioul) a amené cet auteur à émettre l'hypothèse d'une "céramisation" des derniers chasseurs - collecteurs castelnoviens. À la suite de cette découverte, plusieurs autres abris-sous-roche du Val d'Adige ont livré des ensembles similaires, entraînant ainsi la création du "groupe de Gaban". 
La recherche présentée ici a consisté en une nouvelle étude des matériaux issus des fouilles Bagolini, notamment par l'analyse de près de 2600 outils retouchés en silex (je tiens d'ailleurs à remercier A. Pedrotti de l'université de Trento, ainsi que M. Lanzinger et G. Dalmeri du Muséum d'histoire naturelle qui ont rendu possible cette étude). Pour commencer, il m'a semblé indispensable de procéder à une nouvelle analyse de la stratigraphie de l'abri elle-même. La consultation des notes de terrain manuscrites du fouilleur montre que la technique de fouilles employée ne permet pas de considérer les résultats obtenus comme fiables (décapages horizontaux arbitraires de $10 \mathrm{~cm}$ d'épaisseur, sans tenir compte des variations sédimentaires d'ailleurs difficilement lisibles sur le terrain), notamment si on les confronte à la répartition verticale des silex. Ainsi, l'identification de couches "mixtes" ne correspond pas à la réalité de la stratigraphie mais simplement à une technique de fouilles peu adéquate entraînant le mélange de diverses occupations. Plus encore, les autres gisements où cette phase de transition serait également attestée s'avèrent avoir été exploités selon cette même technique de fouille. En conséquence, l'hypothèse d'une acculturation des groupes mésolithiques par les premiers paysans de la plaine padane ou du Frioul perd là tous ses arguments stratigraphiques.

Il n'en reste pas moins vrai que les industries lithiques de tous ces groupes du Néolithique ancien padan (groupe de Gaban, groupe de Vhò, Fiorano, groupe de Fagnigola, groupe de Sammardenchia) montrent des caractères qui semblent proches de ceux du Castelnovien.

\section{Les industries lithiques des premiers paysans de l'Ita- lie du Nord-Est}

Vérifier la véracité de cette similitude des industries entre les derniers chasseurs et les premiers paysans m'a demandé de revoir chacun de ces groupes. L'analyse des industries lithiques selon une approche techno-économique est en effet encore relativement peu développée en Italie septentrionale, les études étant en général limitées à une analyse typologique. J'ai donc choisi de réétudier un gisement si possible caractéristique de chacun des groupes culturels du premier Néolithique nord italien (à l'exception du groupe de Vhò dont l'industrie ne se différencie a priori pas de celle du Fiorano). Pour le Castelnovien, le choix s'est porté sur les niveaux inférieurs de l'abri Gaban. Pour le groupe de Gaban lui-même, en plus du site éponyme, j'ai également analysé les séries du site de plein air de La Vela ( ${ }^{\circ} 269$ sur la carte). Pour le Fiorano, j'ai pu accéder aux industries d'une des occupations du grand gisement de Lugo di Grezzana $\left(n^{\circ} 273\right)$. Enfin, pour les groupes du Frioul, j'ai eu accès aux deux gisements éponymes: Fagnigola $\left(\mathrm{n}^{\circ} 253\right)$ et Sammardenchia ( $\left.{ }^{\circ} 254\right)$.

L'analyse de ces industries visait à retrouver les schémas opératoires, les séquences conceptuelles, à l'origine des productions réalisées. Ce type de recherche permet d'identifier les choix techniques effectués par les tailleurs, les préférences opératoires choisies entre plusieurs possibles. C'est à ce niveau là d'analyse que l'on peut identifier des traditions culturelles.

Ainsi, il apparaît tout d'abord que le Fiorano et le groupe de Gaban possèdent des schémas opératoires très proches sinon identiques. Seule change la gamme d'outils, puisque le groupe de Gaban montre un taux d'armatures beaucoup plus élevé que le Fiorano. On ne peut exclure l'existence d'une complémentarité entre gisements, puisque les sites Fiorano se situent dans la plaine padane, où l'agriculture peut être aisément développée, alors que le groupe de Gaban se développe en milieu de montagne et peut donc correspondre à une spécialisation de ces occupations vers des activités cynégétiques. Le groupe de Gaban pourrait donc correspondre en réalité à des occupations plus ou moins temporaires (saisonnières) de paysans du Fiorano, spécialisées vers la chasse. Malheureusement, force est de constater l'absence d'analyse des faunes consommées qui pourraient aider à valider cette hypothèse.

Pour les groupes du Frioul, l'analyse conjointe des industries, des données de fouilles et des datations radiocarbone montre que ces faciès ne peuvent être considérés comme homogènes. Au contraire, on distingue nettement deux phases d'occupations sur les gisements. La première se place entre 5600 et 5400 environ, c'est-à-dire antérieurement à tous les sites du Néolithique ancien de la plaine padane, et comporte des industries spécifiques, que l'on ne retrouvera guère ultérieurement, mais qui sont par contre attestées dans les sites castelnoviens. Une seconde phase vient se placer entre 5200 et 4600 , et présente des schémas opératoires semblables aux groupes contemporains du Néolithique de la plaine padane, ainsi que des types d'outils qui leur sont spécifiques. On considère d'ailleurs généralement, avec raison, que ces types d'outils particuliers (burins de Ripabianca et rhomboïdes) témoignent de l'ancrage

Données sur les axes 1 et 2 (099\%)

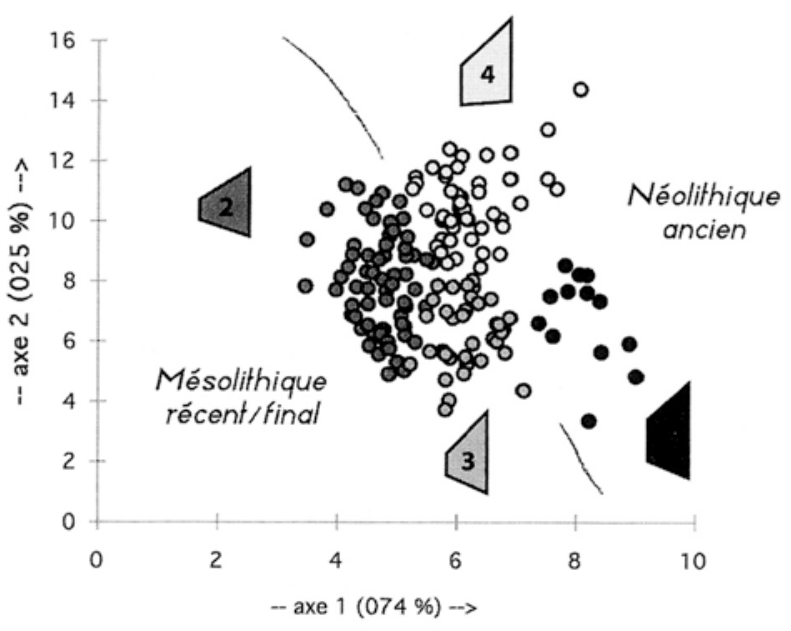

Fig. 2 - Les armatures de flèches en silex des divers groupes humains impliqués dans la néolithisation de l'Italie du Nord ont toutes une forme trapézoïdale obtenue selon des procédés techniques identiques. Il est alors très difficile de discerner des critères distinctifs entre les pièces produites par les derniers chasseurs mésolithiques et celles des premiers paysans néolithiques. En tenant compte de diverses mensurations de ces trapèzes (angle grande base/grande troncature, indice de symétrie, indice d'allongement) et en les analysant d'un point de vue statistique, il devient possible d'opérer cette distinction. La figure montre ainsi les résultats de l'analyse factorielle discriminante réalisée sur les données issues d'une classification en nuées dynamiques, qui permet de distinguer quatre types de trapèzes, et illustrant ainsi une évolution vers des formes plus allongées et plus asymétriques avec le Néolithique ancien. 
Hypothèse 1 : acculturation de chasseurs collecteurs mésolithiques

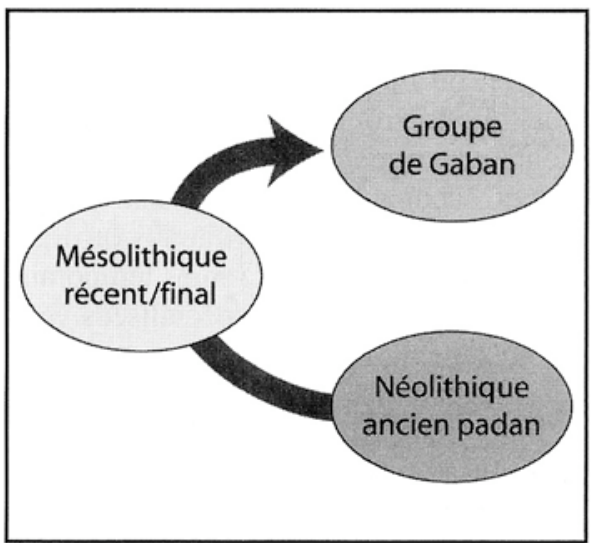

Hypothèse 2 : assimilation de traits mésolithiques par les groupes d'agriculteurs éleveurs néolithiques

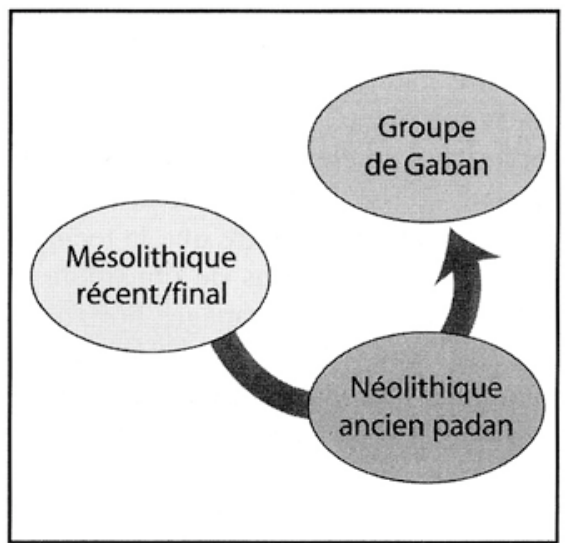

Hypothèse 3 : colonisation en deux temps depuis une même zone géographique extérieure

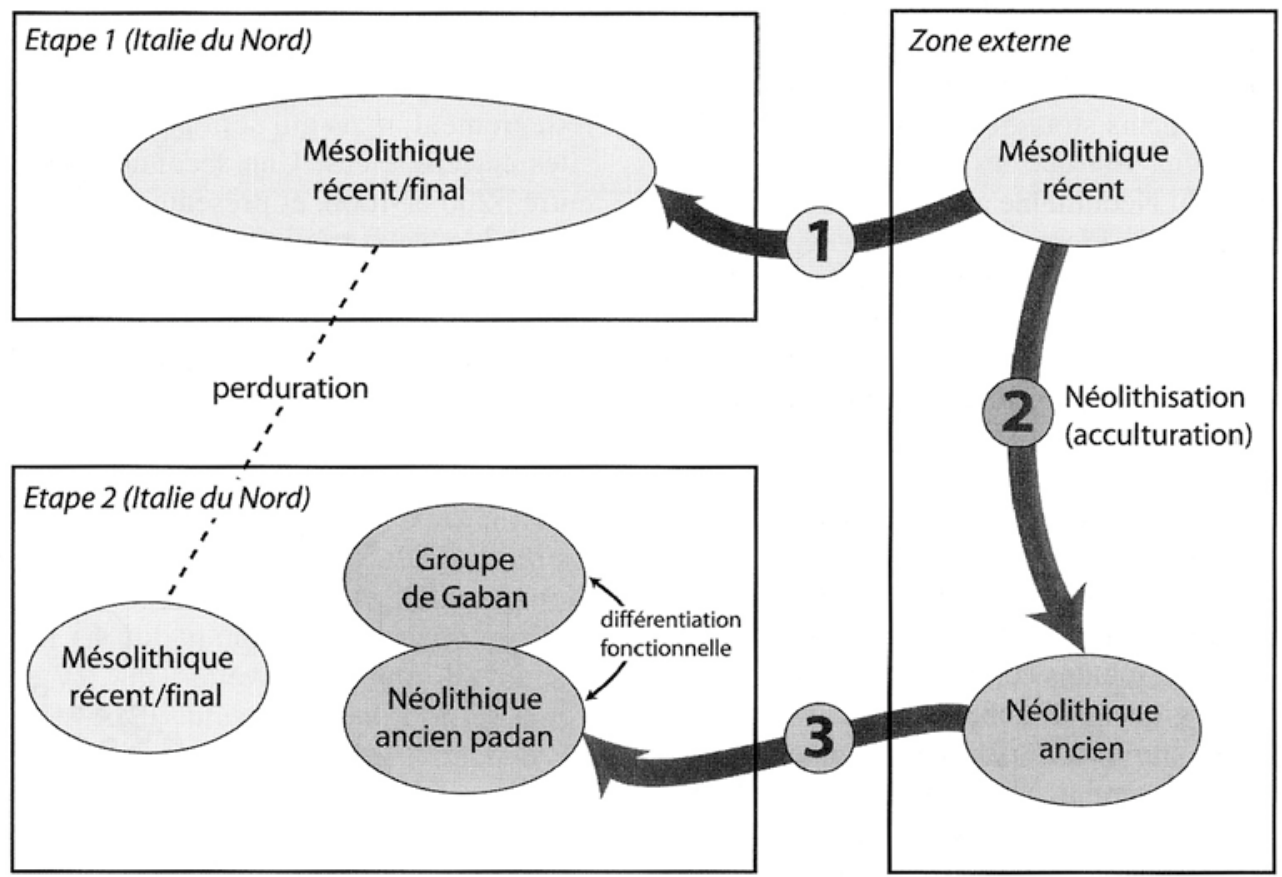

Fig. 3 - Schémas synthétiques des différentes hypothèses explicatives de la formation du groupe de Gaban. Les deux premières ne rendent qu'imparfaitement compte des similarités et différences entre les divers groupes humains impliqués dans les processus de néolithisation de cette région. La troisième hypothèse me paraît mieux rendre compte de la situation observée.

des groupes du Néolithique padan dans les traditions de la Ceramica impressa adriatica.

Le Mésolithique castelnovien montre lui aussi des schémas opératoires proches de ceux des groupes du Néolithique ancien du Nord-Est italien. Les modalités techniques choisies par les tailleurs mésolithiques ne varient pas de façon significative par rapport à celles des tailleurs du Néolithique ancien. La production est par contre évidemment plus orientée vers l'obtention d'armatures suite au fort besoin d'armes de chasse. Si une approche globale de ces armatures, de forme trapézoïdale, ne semblait pas montrer de différences évidentes avec celles du Néolithique ancien, des analyses statistiques permettent de dégager l'existence d'une tendance évolutive, valable au moins au sein de l'abri Gaban (fig. 2). Enfin, l'analyse des datations du Castelnovien indique qu'elles se placent sur l'ensemble du VII ${ }^{\mathrm{e}}$ millénaire et qu'il n'existe pas de dates fiables ultérieures à 6000 avant notre ère. Or, l'hypothèse de l'acculturation d'un groupe par un autre impose la coexistence chronologique de ces deux ensembles. En l'état actuel des datations radiocarbone, il n'existe pas de recoupement entre les niveaux mésolithiques et néolithiques, mais au contraire un hiatus chronologique d'un minimum de quatre siècles entre les 
deux. Comment alors expliquer la similitude indéniable (même s'il existe des évolutions) entre tous ces groupes culturels, qu'ils soient mésolithiques ou néolithiques?

\section{Vers un nouveau modèle de la néolithisation de l'Italie du Nord}

En excluant l'hypothèse difficilement défendable d'une coïncidence, il me semble que le scénario explicatif le plus convaincant est celui d'une colonisation du NordEst italien en deux temps depuis une zone géographique externe, à situer probablement quelque part au long de la côte est adriatique (fig. 3).

Cette zone géographique externe serait occupée, au $\mathrm{VI}^{\mathrm{e}}$ millénaire, par le Mésolithique récent castelnovien. Dans un premier temps, ce Castelnovien diffuserait vers l'Italie septentrionale où il présenterait donc des schémas opératoires identiques à ceux de la zone d'origine. Puis, cette zone originelle subirait, au cours du $\mathrm{VI}^{\mathrm{e}}$ millénaire, des processus de néolithisation par acculturation. Elle garderait alors des schémas opératoires proches de ceux du Mésolithique récent castelnovien, tout en acquérant des traits néolithiques. Ce nouveau Néolithique ancien coloniserait ensuite le Frioul pour y constituer la première phase des groupes de Fagnigola et de Sammardenchia. Dans un second temps, cette colonisation se poursuivrait vers la plaine padane où sa rencontre avec les groupes liés à la montée le long des côtes ouest de l'Adriatique du Néolithique ancien de la Ceramica impressa adriatica formerait le Fiorano et le groupe de Vhò. La spécialisation fonctionnelle de certains gisements Fiorano, orientés vers la chasse, serait à l'origine du groupe de Gaban. De même, la poursuite de la montée des influx de l'Impressa jusque dans le Frioul ferait évoluer les faciès de Fagnigola et Sammardenchia vers leur seconde phase.

La similarité de tous ces groupes culturels avec le Mésolithique récent castelnovien, dont ils se différencient malgré tout par des caractères discrets, serait donc liée à une origine commune. La vérification de ce modèle demande d'affiner nos connaissances sur les groupes du Néolithique ancien du Nord de l'Italie, mais également de toute la frange adriatique, que ce soit du côté occidental où l'on ne connaît guère les industries de l'Impressa, que du côté oriental où les connaissances sont là aussi très limitées. Ces observations constituent ainsi les bases d'un projet à plus long terme, à l'échelle de l'Europe méditerranéenne, visant à améliorer notre compréhension de ces phénomènes complexes qui ont amené l'Homme a quitter ses "habits" millénaires de chasseurs - collecteurs pour endosser ceux que nous portons encore actuellement.

\section{RÉFÉRENCES BIBLIOGRAPHIQUES}

AMMERMAN A.J., CAVALLI SFORZA L.L. (1971) - Measuring the rate of spread of early farming in Europe, Man, 6, p. 674-688.

BAGOLINI B., BIAGI P. (1988) - The first neolithic chipped stone assemblages of northern Italy, in J. et S. Kozlowski dir., Chipped stone industries of the early farming cultures in Europe, Archeologia Interregionalis, Warsaw University, p. 423-448.

CAUVIN J. (1994) - Naissance des divinités, naissance de l'agriculture. La révolution des symboles au Néolithique, Empreintes de l'Homme, CNRS Éditions, Paris, 304 p.

GUILAINE J. (2000) - De l'Orient à l'Occident : la néolithisation de la Méditerranée, questions ouvertes, in A. Pessina A. et G. Muscio dir., La neolitizzazione tra oriente e occidente. Atti del Convegno di Studi, Udine, 23-24 aprile 1999, éd. del Museo Friulano di Storia Naturale, Comune di Udine, p. 11-21.

JUAN CABANILLES J. (1990) - Substrat épipaléolithique et néolithisation en Espagne : apport des industries lithiques à l'identification des traditions culturelles, in D. Cahen et M. Otte dir., Rubané et Cardial, Actes du Colloque de Liège, nov. 1988, ERAUL, n 39, Liège, p. $417-$ 435.

MARCHAND G. (2001) - Les traditions techniques du Mésolithique final dans le Sud du Portugal: les industries lithiques des amas coquilliers de Várzea da Mó et de Cabeço do Rebolador (fouilles M. Heleno), Revista Portuguesa de Arqueologia, vol. $4 \mathrm{n}^{\circ} 2$, p. 47 110.

MAZURIE DE KEROUALIN K. (2003) - Genèse et diffusion de l'agriculture en Europe. Agriculteurs - chasseurs - pasteurs, coll. des Hespérides, éd. Errance, Paris, 184 p.

PERRIN T. (2003) - Mesolithic and Neolithic cultures co-existing in the upper Rhône valley, Antiquity, vol. 77, fasc. 4, p. 745-752.

ZVELEBIL M. (2000) - Les derniers chasseurs-collecteurs d'Europe tempérée, in : A. Richard et C. Cupillard dir., Les derniers chasseurscueilleurs d'Europe occidentale (13000 - 5500 av. J.-C.), actes du Colloque International de Besançon (Doubs, France), 23-25 octobre 1998, Presses Universitaires Franc-Comtoises, coll. Annales Littéraires, p. 379-406.

Thomas PERRIN

Centre d'Anthropologie

39, allées Jules Guesde, 31000 Toulouse

tperrin@free.fr 